\title{
Presenting the Multi-Objective Optimization Model of Search and Rescue Network
}

\author{
Md Mashum Billal \\ Department of Mechanical Engineering \\ University of Alberta \\ Edmonton, Canada
}

\author{
Maryam Maleki \\ Department of Systems Engineering \\ University of Arkansas at Little Rock \\ Little Rock, AR, USA
}

\begin{abstract}
The Search and Rescue Network (SAR) is a kind of emergency network that pursuit people in need or imminent danger. This paper aims using a priori optimization to demonstrate the optimal assignment of HFDF receivers to the Generalized Search and Rescue (GSAR) network, which is independent of the weighting of the transmitter areas. The mathematical model seeks two objectives, the first one is maximizing the expected number of LOBs for HFDF receivers. The second is providing a fair share number of HFDF receivers allowed to cover the frequency. The result shown the efficiency of presented model ran by CPLEX toolbox of MATLAB 2020 software.
\end{abstract}

Keywords: Search and Rescue Network; Priori Optimization; MATLAB 2020 Software; HFDF Receivers.

\section{INTRODUCTION}

Search and rescue network has different forms and each of them with unique risks and dangers to victim and responder [1], [2].The U.S. is founded and maintained a system of search and rescue (SAR) stations encompassing seas and oceans, these stations are responsible for receiving and processing signals from distressed ships, vessels and airplanes in order to initiate the emergency operations. The spark of any emergencies is the time when three or more stations receive and process the same distress signal since in order to find an approximation of distressed vessel three stations are required.

There are many optimization method for solving the search and rescue network. Among them, in this paper, we are planning to apply the multi-objective linear programming (MOLP) which is proposed by [5] to solve the problem as we will define in section 2. To solve their model, [5] convert the model to linear model in order to get the result in a fastetst time.

Simulation is other technique that some reasecrhers used in their studioes to find an answer near to the optimal result [6] [3]. Simulation optimization can be defined as finding the best input variable values of all options, and not evaluating each option explicitly. The objective of simulation optimisation is to minimize the resources spent in a simulation experiment while maximizing data. [6] used simulation technique to reduce the cost of production and the rate of energy waste during the transmission on electricity distribution systems. [3] applied a discrete event simulation approach and scenario discussion to encompass a set of operational decisions to manage the complexity of the system. Moreover, they employed the Arena simulation software for designing blood supply chain to provide a critical comparison of the two primary Key Performance Indicators shortage and outdated units of the BSC.

\subsection{Relation Between Receiving} \section{Subsystems (RS) and Central Control (CC)}

It is noted that each station in the SAR network has only one RS system, but the number of high frequency direction finding (HFDF) receivers in each station is different. In our problem, the number of HFDF receivers varies between 0 and 10. For more clarification, RS probes the entire frequency spectrum and has less sensitive and accurate than the HFDF. Moreover, RS has the limitation on small signal-to-noise ration unlike
HFDF. Every HFDF receivers is allotted to a $1 \mathrm{MHz}$ bund within the frequency spectrum.

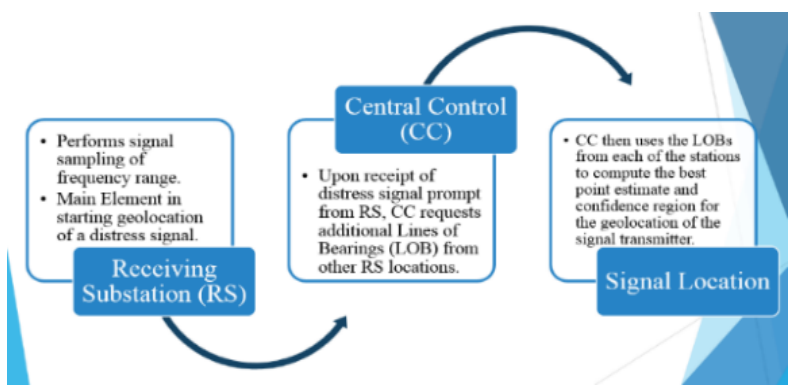

Figure. 1 Relationship between RS and CC

Prior to research background, the following schematic figure depicts the area of research in accordance with the receiving substation and estimated point of transmitted point and an acceptable circularized error radius.

\subsection{Error Radius}

The most recent research history on the topic of search and rescue culminated in optimal methods for the location of stations and frequency assignments. Since the subject is a wellacknowledged area of research, many previous researches had added to the body of knowledge. [4] thoroughly discussed the problem and different analytical approaches. He explained that "classical sensitivity analysis and tolerance analysis were used to analyze the frequency assignments generated by the different weight sequences. The weight sequence with all weights having equal value produced the most robust frequency assignments for all time blocks".

We followed the same footpath to recalculate the results once more time. Although [4] used ADBASE, LINGO and CPLEX IBM ILOG Studio also provided the same computer runs results, which are enclosed to this report as well.

As by [4] cited, the basis of his research is founded mainly on two antecedences, first, Steppe used a two-stage, network-flow multi-Objective linear integer programming (MOLIP) model [5] to determine the optimal position of the stations for the SAR problem. Second, Johnson's further work in this field has established optimal frequency assignments using the MOLIP network-flow model. 


\subsection{Research Objectives}

The goal of this research is to use a priori optimization to show that the optimal assignment of HFDF receivers to the Generalized Search and Rescue (GSAR) network is independent of the weighting of the transmitter areas. This is achieved by examining the impact of changing the weight value of a specific transmitter area on the geolocation likelihood for that area. The mathematical model has two purposes, the first of which is to optimize the predicted number of LOBs for HFDF receivers. And the second is to have a reasonable share of the number of HFDF receivers allowed to cover the frequency [5].

\section{MODEL FORMULATION}

This section is an overview of the multi-objective linear programming (MOLP) and network programming formulas for the search and rescue network [6], [7], [2]. The weights for transmitter areas shall be given by the Department of Defense (DOD).

The notations, parameters, and variables are:

$i$ : transmitter locations

$j$ : receiving locations

$k$ : frequency bands

$F_{i k}$ : Probability of a distress signal from location $i$ on frequency $k$

$P_{i j k}$ : Probability that a distress signal from location $i$ on frequency $k$ is acquired by station $j$

$W_{i j}$ : Probability that a line of bearing from station $j$ is within the acceptable circularized error region defined for location $i$

$U_{i}$ : The normalized weight ( $0-1$ range) of a distress signal from location $i$

$T N$ : The total number of HFDF receivers

FS: The fair share of HFDF receivers for each frequency

Where FS is the integer greater than or equal to the total number of HFDF receivers divided by the total number off frequencies to be covered.

$X_{j k}=1$, if station $j$ is assigned cover frequency $k$, otherwise 0

$Y_{k}=n Y_{k}=\left\{\begin{array}{l}n \\ 0\end{array}\right.$,if frequency $k$ has excess coverage by $n$ stations, otherwise 0

\subsection{Objectives and Constraints}

The model formulation for this multi-objective optimization model of a search and rescue network takes the following form [5]:

Objective Function 1: This objective function maximizes the estimated number of accurate bearing lines for HFDF receivers [2].

$$
\operatorname{Max} \sum_{i} \sum_{j} \sum_{k} U_{i} W_{i j} F_{i k} P_{i j k} X_{j k}
$$

Objective Function 2: This objective function minimizes the excess coverage of HFDF receivers for each frequency.

$$
\operatorname{Min} \sum_{k} Y_{k}
$$

Constraint 1: Limit the number of HFDF frequency assignments at each station to the number of receivers located at each station.

$$
\sum_{k} X_{j k} \leq m_{j}, \quad \forall j
$$

Constraint 2: This restriction allows at least two HFDF receivers to be allocated to cover each frequency.

$$
\sum_{j} X_{j k} \geq 2, \quad \forall k
$$

Constraint 3: Determines the sum of excess coverage provided at each frequency. The vector $Y_{k}$ is the indicator of excess coverage.

$$
\sum_{j} X_{j k}-Y_{k} \leq F S, \quad \forall k
$$

\subsection{Obtained Data from DOD}

A case study of actual data is provided, and the results are regenerated since the software changed. The following data is used to calculate the weights for the problem. Table 1 provides the probability of a signal being transmitted by a transmitter $i$ on frequency $k$.

Table 1. Signal transmission and frequency probability

\begin{tabular}{llll}
\hline$i / k$ & Frequency 1 & Frequency 2 & Frequency 3 \\
\hline Transmitter 1 & 0.04 & 0.04 & 0.04 \\
Transmitter 2 & 0.00 & 0.00 & 0.01 \\
Transmitter 3 & 0.03 & 0.05 & 0.05 \\
Transmitter 4 & 0.00 & 0.00 & 0.00 \\
\hline
\end{tabular}

\begin{tabular}{|c|c|c|c|c|c|c|c|c|c|c|}
\hline \multirow[t]{2}{*}{$j$} & \multicolumn{3}{|c|}{ Transmitter } & \multicolumn{4}{|c|}{ Transmitter } & \multicolumn{3}{|c|}{ Transmitter } \\
\hline & 1 & 2 & 4 & 1 & 2 & 3 & 4 & 12 & 3 & 4 \\
\hline 1 & 0.98 & 0.32 & 0.510 .01 & 10.95 & 0.13 & 30.35 & 50.0 & 0.960 .33 & 30.52 & 20.01 \\
\hline 2 & 0.98 & 0.44 & 0.130 .01 & 10.98 & 80.08 & 80.01 & 10.0 & 0.980 .30 & 00.01 & 10.01 \\
\hline 3 & 0.97 & 0.01 & 0.010 .01 & 10.92 & 20.46 & 60.71 & 10.0 & 0.830 .31 & 10.51 & 10.01 \\
\hline 4 & 0.97 & 0.97 & 0.010 .01 & 10.98 & 80.01 & 10.12 & 20.0 & 0.900 .01 & & 10.01 \\
\hline 5 & 0.98 & 0.03 & 0.010 .01 & 10.94 & 40.04 & 40.01 & 10.0 & 0.940 .19 & 90.00 & 00.01 \\
\hline
\end{tabular}

Table 2 indicates the likelihood of a signal being transmitted from transmitter $i$ to frequency $k$ and acquired by station $j$.

Table 2. Probability of signal transmission and station acquisition

Table 3 indicates the likelihood that station $\mathrm{j}$ will receive a signal from the transmitter I when a signal has been transmitted. 
Table 3. Probability of station receipt of signal

\begin{tabular}{llllll}
\hline$i / j$ & Station 1 & Station 2 & \multicolumn{2}{c}{ Station 3 Station 4 Station 5 } \\
\hline Transmitter & 0.3808 & 0.747 & 0.1951 & 0.121 & 0.7956 \\
Transmitter & 0.1477 & 0.1301 & 0.1140 & 0.0596 & 0.2504 \\
Transmitter & 0.1471 & 0.0892 & 0.1580 & 0.0834 & 0.1509 \\
Transmitter & 0.0515 & 0.7679 & 0.0615 & 0.0820 & 0.0427 \\
\hline
\end{tabular}

Table 4 offers different weighting sequences for the nine solutions to the sample problem.

Table 4. Weighting sequence for the nine solutions to the sample problem

\begin{tabular}{cccccccccccc}
\hline i/ & Station & \multicolumn{2}{l}{ Station } & Station & Station & Station & Station & \multicolumn{2}{l}{ Station } & Station & Station \\
\hline 1 & 0.25 & 0.50 & 0.167 & 0.167 & 0.167 & 0.70 & 0.10 & 0.10 & 0.10 \\
2 & 0.25 & 0.167 & 0.50 & 0.167 & 0.167 & 0.10 & 0.70 & 0.10 & 0.10 \\
3 & 0.25 & 0.167 & 0.167 & 0.50 & 0.167 & 0.10 & 0.10 & 0.70 & 0.10 \\
4 & 0.25 & 0.167 & 0.167 & 0.167 & 0.50 & 0.10 & 0.10 & 0.10 & 0.70 \\
\hline
\end{tabular}

Table 5. Manual sensitivity analysis range for time block one weight

\begin{tabular}{llll}
\hline Weight \# & Original Value & Low Value & High Value \\
\hline 20 & 0.203 & $1 \%$ & $10 \%$ \\
22 & 0.145 & $16 \%$ & $4 \%$ \\
27 & 0.203 & $6 \%$ & $0 \%$ \\
30 & 0.145 & $28 \%$ & $5 \%$ \\
31 & 0.203 & $11 \%$ & $8 \%$ \\
\hline
\end{tabular}

Table 6. Manual sensitivity analysis range for time block six weights

\begin{tabular}{llll}
\hline Weight \# & Original Value & Low Value & High Value \\
\hline 9 & 0.1491 & $10 \%$ & $15 \%$ \\
20 & 0.1491 & $23 \%$ & $4 \%$ \\
27 & 0.1491 & $3 \%$ & $27 \%$ \\
30 & 0.1355 & $28 \%$ & $4 \%$ \\
31 & 0.1897 & $11 \%$ & $14 \%$ \\
40 & 0.1355 & $2 \%$ & $13 \%$ \\
\hline
\end{tabular}

\subsection{Methodology}

The technique used was a constraint reduced feasible region method in a "toy problem" type of scenario where a condensed version of the larger problem was extracted and run to show that the calculations are accurate, and that the solution is viable.

The constraint reduced method to solve a MCLP is to "convert one of the two criterion functions, in this case $f_{2}(x)$, into a constraint, which is added to the existing constraint set $x \in X$." [6], [8], [9], [10]. The formulation of our toy problem therefore goes from the following objective function and constraint function notation:

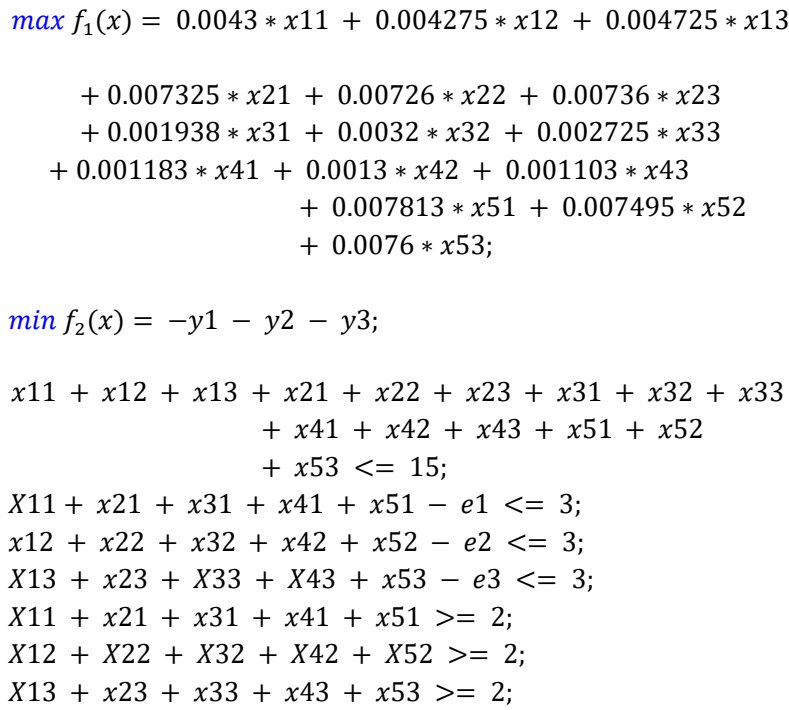

To the following form:

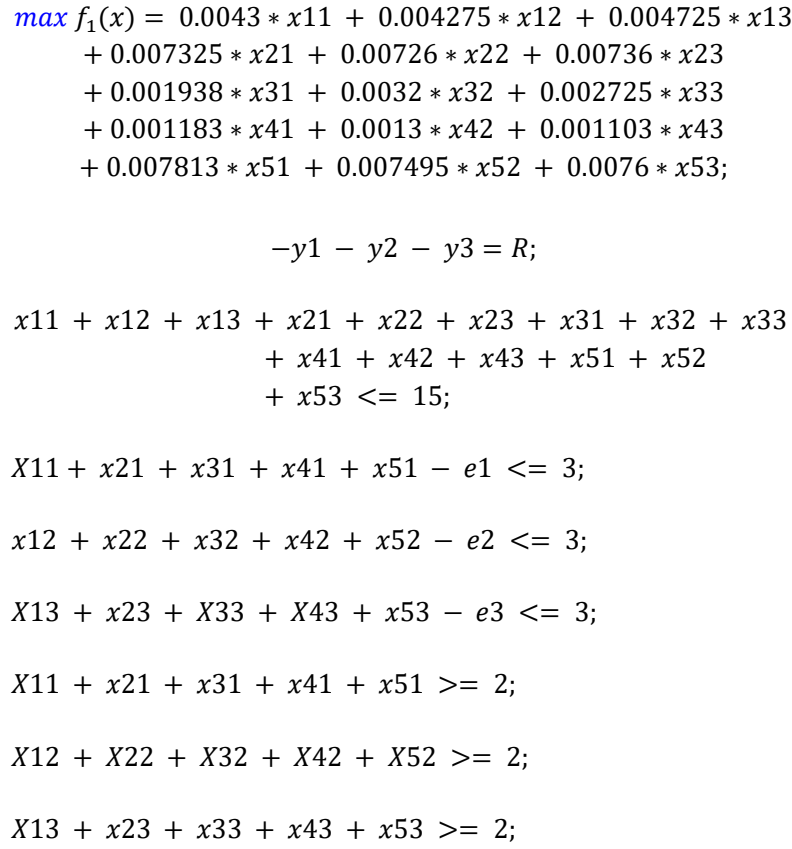

Where $R$ is a "satisficing level for $f_{2}$ ". Then, "by graphically [and numerically] minimizing and maximizing $f_{2}$ over $X$, the feasible region defined by the original constraint set, we are able to find all the $N$-points.

The formulation of the linear program limits decision variables, $X_{j k}$ and $Y_{k}$, to integer values. Specifically, $X_{j k}$ must be equal to zero or one, while $Y_{k}$ may take any positive integer value less than or equal to the number of receiving stations on the network.

For this toy problem, we utilized Lindo Systems' software, Lingo, to input and solve this linear problem. The values of $R$ that we used ranged in value from 0 to -6 . The detailed solution to this problem is presented in the solutions section and compared to the results from some of the other previous thesis papers. 


\section{SOLUTION OF CONSTRAINT REDUCED METHOD}

As the solution procedure explained, we were able to run the model and obtain similar results mentioned in the references. In the appendices part, the Lingo program for the constraint reduced method for the toy problem and different values of $\mathrm{R}$, ranging from 0 to -6 , is provided. The $N$-Points acquired from this solution are tabulated in Table 7 and Table 8 below.

Table 7. $N$-points

\begin{tabular}{ccccccccccccccc}
\hline R Values & \multicolumn{11}{c}{ X-space, N-points } \\
\hline$R$ & $X 11$ & $X 12$ & $X 13$ & $X 21$ & $X 22$ & X23 & X31 & X32 & X33 & X41 & X42 & X43 \\
-6 & 1 & 1 & 1 & 1 & 1 & 1 & 1 & 1 & 1 & 1 & 1 & 1 \\
-5 & 1 & 1 & 1 & 1 & 1 & 1 & 1 & 1 & 1 & 1 & 1 & 0 \\
-4 & 1 & 1 & 1 & 1 & 1 & 1 & 1 & 1 & 1 & 0 & 1 & 0 \\
-3 & 1 & 1 & 1 & 1 & 1 & 1 & 1 & 1 & 1 & 0 & 0 & 0 \\
-2 & 1 & 1 & 1 & 1 & 1 & 1 & 0 & 1 & 1 & 0 & 0 & 0 \\
-1 & 1 & 1 & 1 & 1 & 1 & 1 & 0 & 1 & 0 & 0 & 0 & 0 \\
0 & 1 & 1 & 1 & 1 & 1 & 1 & 0 & 0 & 0 & 0 & 0 & 0 \\
\hline
\end{tabular}

Table 8. $N$-points continued

\begin{tabular}{|c|c|c|c|c|c|c|c|c|c|c|}
\hline \multicolumn{9}{|c|}{$\mathrm{X}$-space, N-points Continued } & \multicolumn{2}{|c|}{ Y-space, N-Points } \\
\hline $\mathrm{X} 51$ & $\mathrm{X} 52$ & X53 & Y1 & $\mathrm{Y} 2$ & Y3 & E1 & E2 & E3 & $\mathrm{f}_{1}(\mathrm{x})$ & $\mathrm{f}_{2}(\mathrm{x})$ \\
\hline 1 & 1 & 1 & 0 & 0 & 0 & 2 & 2 & 2 & 0.069601 & -6 \\
\hline 1 & 1 & 1 & 0 & 0 & 0 & 2 & 2 & 1 & 0.068498 & -5 \\
\hline 1 & 1 & 1 & 0 & 0 & 0 & 1 & 2 & 1 & 0.067315 & -4 \\
\hline 1 & 1 & 1 & 0 & 0 & 0 & 1 & 1 & 1 & 0.066015 & -3 \\
\hline 1 & 1 & 1 & 0 & 0 & 0 & 0 & 1 & 1 & 0.064077 & -2 \\
\hline 1 & 1 & 1 & 0 & 0 & 0 & 0 & 1 & 0 & 0.061352 & -1 \\
\hline 1 & 1 & 1 & 0 & 0 & 0 & 0 & 0 & 0 & 0.058152 & 0 \\
\hline
\end{tabular}

The following solution is gained through the thesis's results, and it is presented that the Lingo's output is closely matched the EVAL computer software developed by DOD.

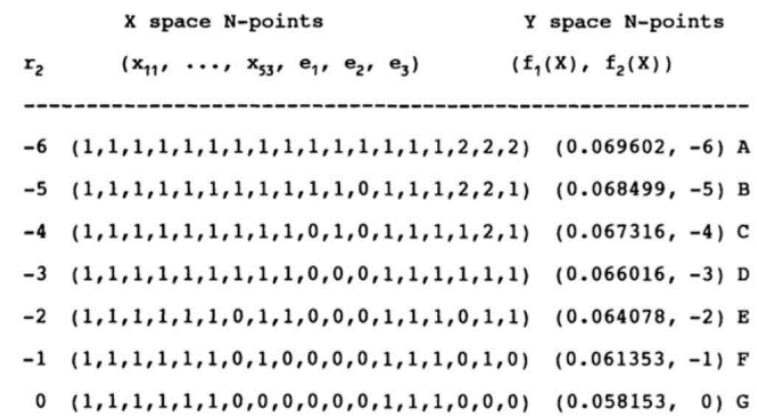

Figure. 2 Solution
The following graphical representation depicts the Y-space which is the optimal values given from the trade-offs between two objective functions.

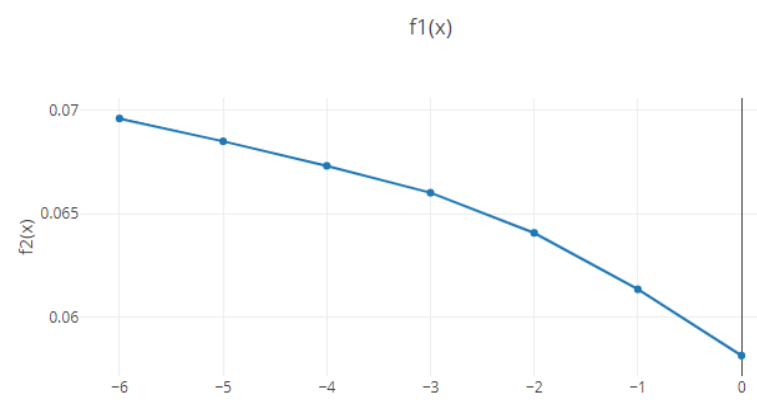

Figure. 2 Graph of $N$-points in $Y$-space demonstrating the efficient frontier

\section{CONCLUSION}

This paper used a priori optimization to demonstrate the optimal assignment of HFDF receivers to the Generalized Search and Rescue (GSAR) network, which is independent of the weighting of the transmitter areas. The model objective presented was to optimize the estimated number of LOBs for HFDF receivers and to provide a reasonable share of the number of HFDF receivers allowed to cover the frequency. Although optimization models are of remarkable importance when it boils down to accuracy, being time consuming and engaging computational resources are the reasons to consider artificial intelligence approaches too, such as Simulated Annealing algorithm [11], Genetic Algorithm [12], [13], [14], discrete event simulation [6] [3], and heuristic algorithms [15][17].

\section{REFERENCE}

[1] R. R. Murphy et al., "Search and Rescue Robotics," in Springer Handbook of Robotics, Berlin, Heidelberg: Springer Berlin Heidelberg, 2008, pp. 1151-1173.

[2] N. Razi and M. Karatas, "A multi-objective model for locating search and rescue boats," Eur. J. Oper. Res., vol. 254, no. 1, pp. 279-293, Oct. 2016, doi: 10.1016/j.ejor.2016.03.026.

[3] M. Arani, X. Liu, and S. Abdolmaleki, "ScenarioBased Simulation Approach for An Integrated Inventory Blood Supply Chain System," in 2020 Winter Simulation Conference (WSC), Dec. 2020, pp. 1348-1359, doi: 10.1109/WSC48552.2020.9384018.

[4] J. C. I. Jr, "An A Priori Multiobjective Optimization Model of a Search and Rescue Network," 1992.

[5] S. Hayat, E. Yanmaz, T. X. Brown, and C. Bettstetter, "Multi-objective UAV path planning for search and rescue," in 2017 IEEE International Conference on Robotics and Automation (ICRA), May 2017, pp. 5569-5574, doi: 10.1109/ICRA.2017.7989656.

[6] M. Arani, M. Dastmard, Z. D. Ebrahimi, M. Momenitabar, and X. Liu, "Optimizing the Total Production and Maintenance Cost of an Integrated Multi-Product Process and Maintenance Planning (IPPMP) Model," in 2020 IEEE International 
Symposium on Systems Engineering (ISSE), Oct. 2020, pp. 1-8, doi: 10.1109/ISSE49799.2020.9272236.

[7] M. MomeniTabar, Z. D. Ebrahimi, S. H. Hosseini, and M. Arani, "A Proposed Lean Distribution System for Solar Power Plants Using Mathematical Modeling and Simulation Technique," 2020, doi: 978-1-7281-9677$0 / 20 / \$ 31.00$.

[8] A. Mehrez, M. Eben-Chaime, and J. Brimberg, "Locational Analyses of Military Intelligence Ground Facilities : EMOR,” MORS, 1996, pp. 61-69.

[9] S. Hayat, E. Yanmaz, C. Bettstetter, and T. X. Brown, "Multi-objective drone path planning for search and rescue with quality-of-service requirements," Auton. Robots, vol. 44, no. 7, pp. 1183-1198, Sep. 2020, doi: 10.1007/s10514-020-09926-9.

[10] I. Nielsen, G. Bocewicz, and S. Saha, "Multi-agent Path Planning Problem Under a Multi-objective Optimization Framework," pp. 5-14, 2021, doi: 10.1007/978-3-030-53829-3_1.

[11] M. Arani, M. Momenitabar, Z. Dehdari Ebrahimi, and X. Liu, "Unrelated Parallel Machine Scheduling Problem Considering Inventories Subject to Resource Constraints and Due Dates," 2021.

[12] M. M. Billal and M. M. Hossain, "Multi-Objective Optimization for Multi-Product Multi-Period Four Echelon Supply Chain Problems Under Uncertainty,"
J. Optim. Ind. Eng., vol. 13, no. 1, pp. 1-17, 2020, doi: 10.22094/joie.2018.555578.1529.

[13] A. S. Abir, I. A. Bhuiyan, M. Arani, and M. M. Billal, "Multi-Objective Optimization for Sustainable Closed-Loop Supply Chain Network Under Demand Uncertainty: A Genetic Algorithm," 2020.

[14] B. Afshar-Nadjafi and M. Arani, "Multimode Preemptive Resource Investment Problem Subject to Due Dates for Activities: Formulation and Solution Procedure," Adv. Oper. Res., vol. 2014, pp. 1-10, 2014, doi: 10.1155/2014/740670.

[15] Y. Chan, J. A. Fowe, and M. Arani, "Routing in a Stochastic Network with Nonrecurrent Incidents: Behavioral Interpretation of Dynamic Traffic Assignment," ASCE-ASME J. Risk Uncertain. Eng. Syst. Part A Civ. Eng., vol. 6, no. 1, p. 4020002, Mar. 2020, doi: 10.1061/AJRUA6.0001033.

[16] M. Arani, M. M. Rezvani, H. Davarikia, and Y. Chan, "Routing of Electric Vehicles in a Stochastic Network with Non-recurrent Incidents," Am. Sci. Res. J. Eng. Technol. Sci., Dec. 2019.

[17] M. Maleki, Y. Chan, and M. Arani, "Impact of Autonomous Vehicle Technology on Long Distance Travel Behavior," IISE Annu. Conf. Expo 2020, Jan. 2021. 